\section{Multifocal high-grade prostatic intraepithelial neoplasia is still a significant risk factor for adenocarcinoma}

\section{John R. Srigley, MD, FRCPC, ${ }^{*}$ Jennifer L. Merrimen, MD, FRCPC; ${ }^{\dagger}$ Glenn Jones, MD, MSc, FRCPC,* Munir Jamal, MD, FRCSC}

We read with great interest the recent CUA guidelines on prostate biopsy methodology. ${ }^{1}$ We note that the recommendation regarding high-grade prostatic intra-epithelial neoplasia (HGPIN) states that "in the current era of extended biopsy schemes, HGPIN is no longer considered a strict indication for repeat biopsy and patients should be followed clinically." ${ }^{11}$

Our Canadian research group, set out to specifically address the issue of HGPIN on prostatic needle biopsy (PNB) after 2 large review articles authored by high profile urological pathologists, rendered differing conclusions regarding the significance of HGPIN on PNB..$^{2,3}$ We used a large Canadian database containing over 12000 patients undergoing initial PNB and concluded that HGPIN, when multifocal, is a significant risk factor for prostate cancer (PCa) carrying an odds ratio (OR) of $1.38 .{ }^{4}$ In fact, the risk generally increases with the extent of HGPIN such that increasing cores involved correlates with increasing risk of PCa on follow-up PNB. ${ }^{4}$ Our initial study, although well-controlled with a benign group and statistically sound, did contain a mixture of prostate sampling protocols, so we recently repeated the study, specifically limiting the analysis to include only extended biopsy protocols yielding 10 or more cores. ${ }^{5}$ On the repeat study, we again found the same results. Patients with multifocal HGPIN carry a significantly increased risk of detecting PCa on follow-up PNB compared to patients with benign findings on initial PNB. ${ }^{5}$ Again, there is a general elevation in risk with increasing volume of HGPIN in the initial sample with 1 core showing no increased risk of PCa (OR 0.68) but 2 cores and $>2$ cores involved by HGPIN showing ORs of 2.57 and 3.61, respectively. ${ }^{5}$ Our studies show that HGPIN, when multifocal, is still a significant independent risk factor for $\mathrm{PCa}$, even in the current era of extended PNB protocols. While it is a sound recommendation that patients with unifocal HGPIN be followed clinically with PSA and DRE, it is our recommendation and practice that patients with multifocal HGPIN should undergo a repeat biopsy within 1 year even in the absence of PSA or DRE changes.

We also suggest that pathologists with expertise in urological pathology be involved in the CUA guideline development and review process, especially when recommendations are being rendered for entities diagnosed solely on the basis of morphological findings.

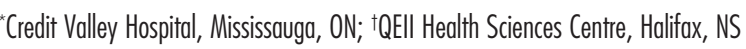

Competing interests: None declared.

This paper has been peer-reviewed.

\section{References}

1. El-Hakim A, Moussa S. CUA guidelines on prostate biopsy methodology. Can Urol Assoc J 2010;6:89-94.

2. Schlesinger C, Bostwick DG, Iczkowski KA. High-grade prostatic intraepithelial neoplasia and atypical small acinar proliferation: predictive value for cancer in current practice. Am I Surg Pathol 2005;29:1201.

3. Epstein JI, Herawi M. Prostate needle biopsies containing prostatic intraepithelial neoplasia or atypical foci suspicious for carcinoma: implications for patient care. J Urol 2006;175:820.
4. Merrimen JL, Jones $G$, Walker $D$, et al. Multifocal high grade prostatic intraepithelial neoplasia is a significant risk factor for prostatic adenocarcinoma. J Urol 2009;182:485.

5. Merrimen JL, Jones G, Srigley JR. Is high grade prostatic intraepithelial neoplasia still a risk factor for adenocarcinoma in the era of extended biopsy sampling? Pathology 2010;42:325-9.

Correspondence: Dr. John R. Srigley, Professor, Department of Pathology and Molecular Medicine, McMaster University, Department of Laboratory Medicine, The Credit Valley Hospital 2200 Eglinton Ave West, Mississauga, 0N L5M 2N1; fax: 905-813-4132; isrigley@cvh.on.ca

\section{A technique using a resectoscope sheath under direct vision and nasogastric tube for difficult catheterization following TURP}

\section{Kenan Isen, $M D$}

Several different techniques under direct vision have been described for the management of difficult ureteral catheterization following transurethral resection of the prostate (TURP). ${ }^{1-3}$ However, all of these tecniques require a specific material (mini ureteroscope or The Peel-away sheath, [Bard, Covington, GA]) which may not available. I would like to describing a simple technique, which does not require special material, to make ureteral catheterization easier following TURP.

After failing an initial attempt to place a Foley urethral catheter, I placed a resectoscope sheath under direct vision into the bladder following TURP. When the resectoscope is inside the bladder, the optical lens and obturator are retrieved from the resectoscope sheath. After that, a nasogastric (NG) tube is advanced through the resectosope sheath into the bladder. When the NG tube is inside the bladder, the distal tip of NG tube is amputated and the resectoscope sheath is withdrawn. The NG tube is fixed to the penis with a standard plaster. The procedure takes about 3 minutes. The NG tube is retrieved 1 or 2 days after the procedure.

The procedure time is minimal. Placing the resectoscope is not technically difficult to maneuver by most urologists. The risk of urethral trauma should be minimized during the procedure. This technique can be performed in any operating room to avoid suprapubic catheterization following TURP.

Department of Urology, Ministry of Health, Diyarbakır Education and Research Hospital, Diyarbakır, Turkey

Competing interests: None declared.

This paper has been peer-reviewed.

\section{References}

1. Villanueva C, Hemstreet GP. Difficult male urethral catheterization: a review of different approaches. Int Braz J Urol 2008:401-11.

2. Rozanski TA, Salazar F, Thompson IM. Direct vision bladder catheterization using a short rigid ureteroscope. Urology 1998;51:827-8.

3. Lowe MA, Defalco AJ. New endourologic technique for catheter placement after TURP, prostatectomy, and difficult urethroscopy. Urology 1992;40:461-3.

Correspondence: Dr. Kenan Isen, Ofis cami sok, Ayylldz Apt., Kat: 5 No:15, Diyarbakır, TR-21 100, Turkey; kenanisen@hotmail.com 